\title{
The holdouts
}

\section{The contrarians of physics get strung out in Chicago.}

\section{George Zebrowski}

$\mathrm{T}$ he annual reunion of string-theory malcontents was held in Chicago on 1 January 2090. Again, the tired theme of the gathering was that real and pseudoscience had somehow reversed roles by the 2050s, with string theory being the ruling dogma of establishment delusionists. The rift among physicists had never been greater or lasted so long. Some science journalists attributed it to the life-extension therapies that had come in during the 2020s.

"Just listen to this," said Chairman ColeBorner at the opening session, reading from a letter of protest sent to the gathering by Delius Feerce: "String theory, despite experimental support, stands blessedly outside our experiment-bound common sense. RandallSundum's so called 'decisive' evidence, the detection of neutrino-warp showers at Cavendish-Lunar 5, was just a pale shadow of the truth of string theory, which rather than needing positive proof is still content to rest on the lack of disproof. Positive-proof seekers tend to find what they are looking for, in the manner of wish-fulfilment. Karl Popper's falsification criterion is more modest - and lack of falsification is what string theory has always had."

"Superstition!" cried Cole-Borner. "They see what they wish and it's consistent with everything!" He laughed loudly. "Listen, there's more rhapsody."

He read: "The theoretician swims free! Einstein knew how the experiments would turn out, and said he would have felt sorry for the Lord if they had failed. He respected experiment, but speculation, hypothesis and guesses at theory are better beginnings. To experiment is to start with poverty. To start with 'free creations' is to start with riches!"
Cole-Borner looked up from the letter and said, "It's strange that they deny the support of Randall and Sundum's gravitational data, which not only appear to confirm string theory but also a broader cosmology. Their collective delusion is a blot on the history of science. It is not so much string theory that we object to, but the method of its confirmation, which is outright assumption. They reject the embarrassment or triumph that science seeks methodically through experimental experience."

At that moment a holo of Great Hawking's downloaded creative persona intruded into the great space above the hall. "All we ever know is our models," the ghost said from his citadel, "but never the reality that may or may not exist behind the models and casts its shadow upon us who are embedded inside it. We imagine and intuit, then point the finger and wait to see which suspect for truth turns and runs. Our models may get closer and closer, but we will never reach direct perception of reality's thing-in-itself."

"Too much science fiction in the science — that's where it all started," muttered ColeBorner as the holo winked out.

It was at once replaced by another invader.

"Only second-rate scientists make fun of science fiction," Sir Arthur C. Clarke said from his download dais. "Why all this blame? We invent whatever it takes to make a theory work. We even guess the truth. The operationalists don't care why a theory works, so the possible untruth of strings is irrelevant. At the very least strings provide a useful framework to organize our facts and observations, and stimulate further insight into nature's appearance as both continuous and particulate.

"Truth? The phenomena laid bare before us, the user's control panel ripped away to

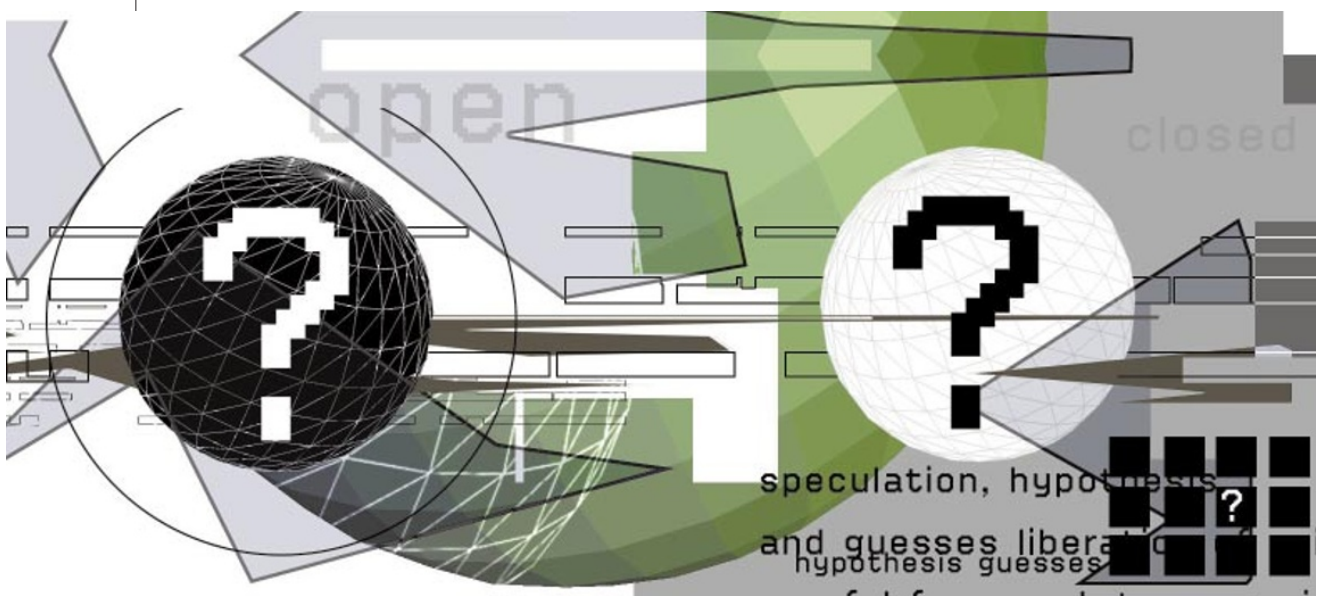

show the hard and soft wares that run it? That event horizon we embedded creatures cannot cross, much less return to tell the tale ... say, there may be a good story in that!"

He smiled, waved, and winked out.

"Very interesting," Cole-Borner muttered. "But there are still some naive realists among us who wonder why our best theories give the appearance of objectivity gained from outside the system rather than from within it."

What troubled the holdouts most was that string theorists had guessed right and worked backwards to the confirmations even deriving relativity from string theory in 2025.

It might all have been a matter of style, of how flexibly the ancient insight of granular atomism was to be expressed - from tiny billiard balls and an absolute void, to clouds of particles, to pastas, loose, circular, and cosmic. It was remarkably suspicious how much could be done by mathematical descriptions from 'inside' reality, based on an imaginative, possibly illusory view from 'outside'.

Today's outgoing vacuum-drive starships, repulsive-force drivers, superluminally entangled communications, teleporters, and superbiologies, suggest the approximate validity of String Regime Unification (SRU) and the Infinite Background-Membrane Cosmology (IBMeC) in which it is expressed, and seems unlikely to be only a coincidental match of theory with a transcendent reality. Close enough but incomplete? We can admit that much to the holdouts, in all Gödelian modesty, as we rejoice in the liberation of science from the tyranny of absolute truth.

Our sciences exist in a sea of ambiguity. Hermann Bondi (if only he had left us his downloaded persona!) reminds us that the power of science comes from being able to say something, without having to say everything. Absolute truth is too exacting for our creative needs, finite beings that we are.

What a difference a century has made: the force that is accelerating universal expansion now drives our electric power generators! One can only wonder at Hawking's desire to know the mind of God. What was he hoping to do with that knowledge? Break into the codes and rewrite reality itself?

The holdouts say no.

But we say yes.

George Zebrowski received the 1999 John W.

Campbell Memorial Award for Best Novel (Brute Orbits, HarperCollins). Cave of Stars (Harper) is his new novel. 